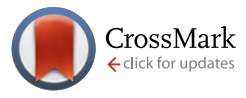

Cite this: Med. Chem. Commun., 2016, 7, 1209

\title{
Design and synthesis of fused soluble epoxide hydrolase/peroxisome proliferator-activated receptor modulators $\dagger$
}

\author{
R. Blöcher, C. Lamers, S. K. Wittmann, O. Diehl, T. Hanke, D. Merk, D. Steinhilber, \\ M. Schubert-Zsilavecz, A. S. Kahnt and E. Proschak*
}

\begin{abstract}
Metabolic syndrome (MetS) is a widespread, complex disease cluster which consists of hypertension, atherosclerosis, dyslipidaemia and type II diabetes. The treatment of MetS requires multiple pharmaceutical agents leading to complex polypharmacy. Multi-target compounds might reduce the number of required drugs in MetS patients. In this study we fused three different pharmacophores of soluble epoxide hydrolase (sEH) inhibitors and peroxisome proliferator-activated receptor (PPAR) agonists. The most promising fused scaffold exhibits multi-target activity and represents a valuable starting point for design and evaluation of fused sEH/PPAR modulators.
\end{abstract}

Received 19th January 2016,
Accepted 10th April 2016
DOI: 10.1039/c6md00042h
www.rsc.org/medchemcomm

\section{Introduction}

Metabolic syndrome (MetS) is a complex disease cluster with high prevalence in the western society. ${ }^{1}$ Central obesity is regarded as the most relevant risk factor which is accompanied by dyslipidaemia, type II diabetes, and hypertension. ${ }^{2}$ This so-called "deadly quartet" is accompanied by diverse complications leading to treatment with multiple pharmacological agents. ${ }^{3}$ Complex treatment regimen, also referred as polypharmacy, cause adverse effects, drug-drug interactions, and ultimately, failure of the therapy. ${ }^{4}$ In this context, multitarget approaches offer a promising strategy to deliver safe and efficient drugs for the treatment of complex disease clusters. ${ }^{5}$ Simultaneous modulation of soluble epoxide hydrolase (sEH) and peroxisome proliferator-activated receptor $\gamma$ (PPAR $\gamma$ ) was shown to exhibit synergistic effects in spontaneously hypertensive obese (SHROB) rats. $^{6}$ In this setting, a combination of the SEH inhibitor tAUCB and the PPAR $\gamma$ agonist rosiglitazone efficiently restored insulin sensitivity, lowered blood pressure, and protected the animals from nephropathy. Hypothetically, beneficial effects of dual sEH/ PPAR $\gamma$ modulation might be enhanced by effective control of plasma lipids, which can be achieved by activation of PPAR $\alpha .^{7}$ Thus, a simultaneous modulation of sEH, PPAR $\gamma$, and PPAR $\alpha$ might be a valuable profile of a multi-target agent for effective and safe MetS therapy.

PPARs are ligand-activated transcription factors which are involved in glucose and lipid homeostasis. PPAR $\gamma$ is targeted

Institute of Pharmaceutical Chemistry, Goethe-University of Frankfurt, Max-vonLaue Str. 9, D-60438 Frankfurt am Main, Germany.

E-mail: proschak@pharmchem.uni-frankfurt.de

$\dagger$ The authors declare no competing interests. by thiazolidinediones (TZDs), also known as insulin sensitizers. Fibrates, which are clinically used for treatment of dyslipidemia are addressing all PPAR subtypes with slight preference for PPAR $\alpha .{ }^{8}$ The role of SEH in cardiovascular disease associated with MetS is well known and has been excessively reviewed. ${ }^{9}$ sEH converts epoxyeicosatrienoic acids (EETs) to the corresponding dihydroxyeicosatrienoic acids (DHETs) and SEH inhibition increases levels of EETs while decreasing levels of DHETs. The resulting increase in the EET/DHET ratio has multiple beneficial effects on the cardiovascular system. EETs are described as agonists of PPAR $\alpha$ and PPAR $\gamma^{10}$ which subsequently promotes the expression of sEH. ${ }^{11}$ Thus, there is a substantial crosstalk between $\mathrm{sEH}$ and PPAR activity supporting the assumption of synergistic targeting of the SEH/PPAR axis. ${ }^{12,13}$

We have previously reported that it is possible to combine the pharmacophores of an SEH inhibitor and PPAR agonist using a combinatorial approach. ${ }^{14}$ In the present study we have explored three different scaffolds on their potential for dual sEH/PPAR modulation.

\section{Results and discussion}

Our first attempt to obtain dual SEH/PPAR modulators was the incorporation of the simplified SEH pharmacophore $N$-cyclohexyl$N^{\prime}$-(iodophenyl)urea (CIU) ${ }^{15}$ into bezafibrate (Fig. 1). Bezafibrate is a pan-PPAR agonist exhibiting $\mathbf{E C}_{50}$ values in the micromolar range. ${ }^{16}$ We decided to remove one of the methyl groups in $\alpha$-position of the carboxylic acid and replaced the amide moiety by a urea residue. The resulting starting point 3c of our SAR study comprises a linker length similar to bezafibrate while $3 d$ has an additional methylene unit in the linker region. 


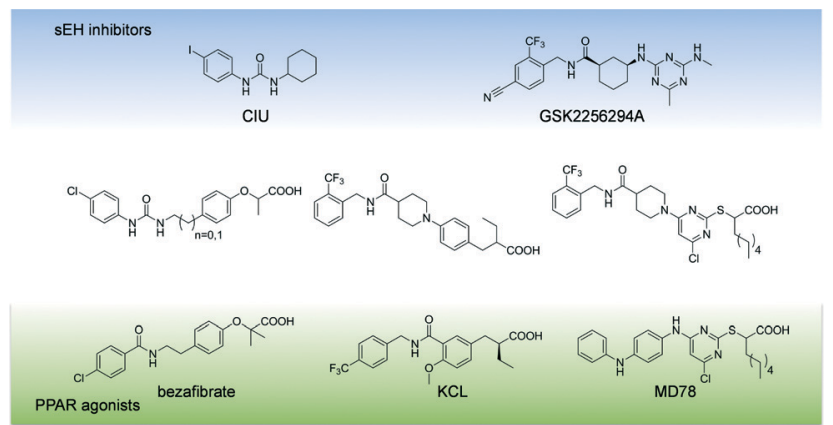

Fig. 1 Scaffolds used for design of fused dual SEH/PPAR modulators.

The synthesis of compounds $3 \mathbf{a}-\mathbf{d}$ was accomplished as shown in Scheme 1. Initially, ethyl 2-bromopropanoate was coupled with the corresponding phenol under Williamson conditions. Then, the nitrile residue was reduced with palladium on carbon as catalyst. The resulting primary amines $2 \mathrm{a}$ and $2 \mathrm{~b}$ were coupled to 4 -chlorophenyl isocyanate to yield ureas $3 \mathbf{a}$ and $\mathbf{3 b}$.

Compounds 3a-d were evaluated in a fluorescence-based assay with recombinant sEH and all are able to inhibit sEH activity with submicromolar potency. Esters $3 \mathbf{a}$ and $\mathbf{3 b}$ were almost one order of magnitude more potent than the corresponding acids due to the hydrophobic nature of the SEH binding site. Furthermore, the ethyl linker was slightly preferred. Unfortunately, none of the compounds was able to activate any of the PPAR subtypes at a concentration of $30 \mu \mathrm{M}$, which forced us to pursue a different scaffold.

We decided to exchange the urea by an amide residue as epoxide mimetic for SEH targeting and chose the $\mathrm{N}$-benzyl amide of isonipecotic acid moiety present in the clinical candidate GSK2256294A that targets $\mathrm{sEH} .{ }^{17} \mathrm{~N}$-Benzylamides are also known as privileged structures in the design of PPAR agonists with different subtype preferences, represented by KCL. ${ }^{18,19}$ The synthesis of the chimera compounds 5a-d was accomplished by isobutyl chloroformate (IBCF) mediated amide coupling of 2-trifluoromethylbenzylamine and 1-(4-formylphenyl)piperidine-4-carboxylic acid. The resulting ethyl cinnamate derivatives 5a and $\mathbf{5 d}$ were obtained under Wittig-Horner conditions followed by reduction with magnesium in methanol and saponification under microwave conditions as described before (Scheme 2).

All derivatives 5a-d exhibited sEH nanomolar inhibition. Since acids and the corresponding esters showed less differences in activity, we assumed that the scaffold was very well-

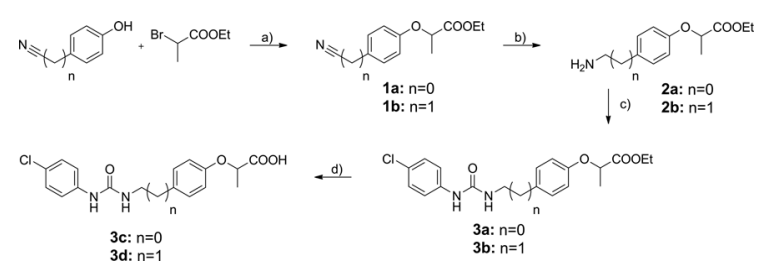

Scheme 1 a) $\mathrm{K}_{2} \mathrm{CO}_{3}$, acetone (abs.), reflux, $12 \mathrm{~h}$, b) $\mathrm{Pd} / \mathrm{C}, \mathrm{AcOH}, \mathrm{EtOH}$ (abs.), $\mathrm{H}_{2}, 12 \mathrm{~h}, \mathrm{c}$ ) 4-chlorophenyl isocyanate, DIPEA, DCM (abs.), Ar, 12 h, d) $\mathrm{KOH}, \mathrm{THF} / \mathrm{H}_{2} \mathrm{O} / \mathrm{MeOH}(1 / 2 / 1), 90^{\circ} \mathrm{C}, \mu \mathrm{w}, 30 \mathrm{~min}$.

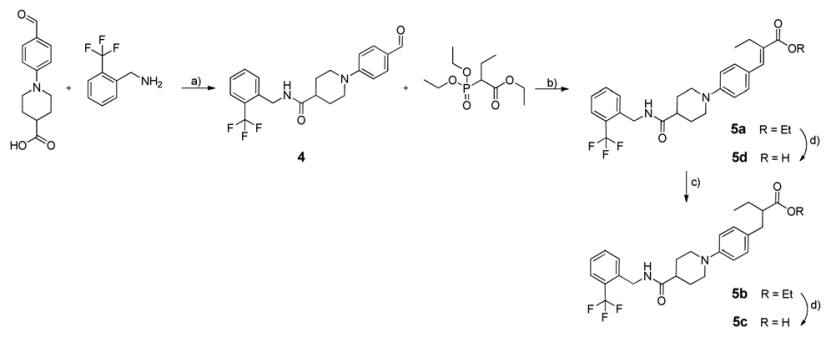

Scheme 2 a) Isobutyl chloroformate (IBCF), trimethylamine (TEA), dichloromethane (DCM) (abs.), Ar, $12 \mathrm{~h}, \mathrm{~b}) \mathrm{NaH}$, tetrahydrofuran (THF) (abs.), $\left.0^{\circ} \mathrm{C}, 2 \mathrm{~h}, \mathrm{c}\right) \mathrm{Mg}, \mathrm{MeOH}$ (abs.), $\left.\mathrm{Ar}, 12 \mathrm{~h}, \mathrm{~d}\right) \mathrm{KOH}, \mathrm{THF} / \mathrm{H}_{2} \mathrm{O} / \mathrm{MeOH}$ (1/2/1), $90{ }^{\circ} \mathrm{C}, \mu \mathrm{w}, 30 \mathrm{~min}$.

tolerated by SEH. However, again no activity on PPARs could be determined. Encouraged by the robust sEH inhibitory activity of $N$-benzyl amides we fused this element with the 4-chloro-2-thio-pyrimidine scaffold which has been described in various studies as a privileged structure for PPAR activation. ${ }^{20}$ The synthesis of compounds $8 \mathbf{c}$ and $8 \mathbf{d}$ is shown in Scheme 3. 2-((4,6-Dichloropyrimidine-2-yl)thio)octanoate was prepared as described previously. ${ }^{21}$ Boc-protected $\gamma$-amino acids were coupled to 2-trifluoromethylbenzylamine mediated by IBCF, followed by Boc cleavage. Scaffold fusion was accomplished under basic conditions in acetonitrile, followed by saponification.

Compounds 8a-d showed sEH inhibitory potency in a low micromolar to submicromolar range. As observed for other scaffolds, acidic derivatives suffered a 4-6-fold loss in potency. Rigidified derivatives $8 \mathbf{a}$ and $8 \mathbf{c}$ were not active on PPARs, while compound 8d partially activated PPAR $\alpha$ and $\operatorname{PPAR} \gamma$ and finally represented the desired triple active agent.

In order to examine PPAR activation under native conditions, mouse fibroblasts were incubated with 8d and a dosedependent adipocyte differentiation could be observed (Fig. 2). Notably the absolute extend of adipocyte differentiation was significantly lower compared to the full agonist

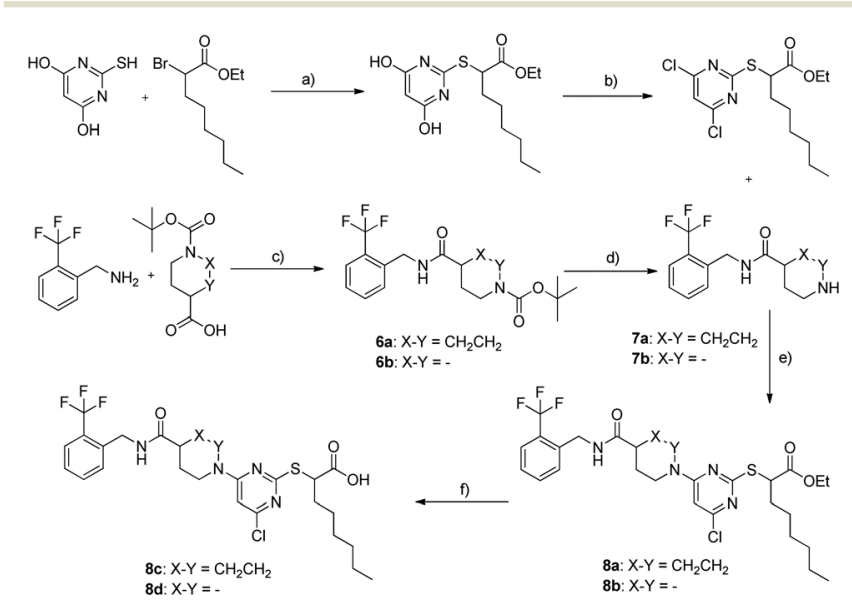

Scheme 3 a) TEA, dimethylformamide (DMF) (abs.), $90{ }^{\circ} \mathrm{C}, 3 \mathrm{~h}, \mathrm{~b}$ ) $\mathrm{POCl}_{3}, \mathrm{~N}, \mathrm{~N}$-diethylaniline, $\left.90{ }^{\circ} \mathrm{C}, 6 \mathrm{~h}, \mathrm{c}\right) \mathrm{IBCF}, \mathrm{TEA}, \mathrm{DCM}$ (abs.), Ar, $12 \mathrm{~h}, \mathrm{~d})$ trifluoroacetic acid (TFA), DCM (abs.), rt, $30 \mathrm{~min}, \mathrm{e})$ TEA, $\mathrm{MeCN}$ (abs.), rt, $12 \mathrm{~h}$, f) $\mathrm{LiOH}, \mathrm{H}_{2} \mathrm{O} / \mathrm{THF}, 45^{\circ} \mathrm{C}, 24 \mathrm{~h}$. 


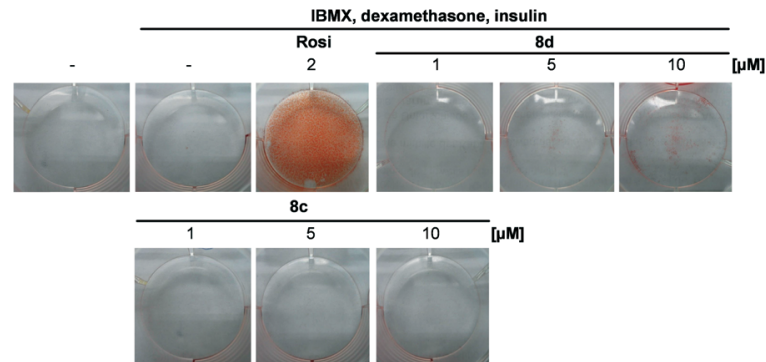

Fig. 2 Oil Red O staining of differentiated murine 3T3-L1 fibroblasts. Staining was performed after 14 days of differentiation. One representative experiment out of 4 is shown. Rosi, rosiglitazone; CIU, sEH inhibitor $N$-cyclohexyl- $N$ '-iodophenyl urea.

rosiglitazone, confirming the favourable partial PPAR agonistic properties of $\mathbf{8 d}$.

An established pharmacophore of dual PPAR agonists consists of an acidic head group, a central aromatic ring, and an additional lipophilic substituent connected to the aromatic ring by a flexible linker. ${ }^{22}$ It is well-established that PPAR ligands should be able to adopt a conformation wrapped around helix $3 .{ }^{23}$ We investigated the differences between $8 \mathbf{c}$ and 8d considering the conformational space. While the flexible linker of 8d allows such conformation, it is almost impossible for the rigid derivative 8c (Fig. 3). With its combined potency on SEH and PPARs, 8d represents a very promising lead to explore the potential of a combined SEH and PPAR modulation as a novel concept to treat MetS and further studies are warranted (Table 1).

\section{Experimental}

General

All starting materials, reagents and solvents were purchased from Alfa-Aesar (Karlsruhe, Germany), Sigma-Aldrich (Hannover, Germany), Apollo Scientific Ltd (Manchester, England), JRD Fluorochemicals, Ltd. (Surrey, England), Axon Medchem BV (Groningen, Netherlands) and used without further purification. TLC was performed using silica coated aluminum foil (particle size $60 \mu \mathrm{m}$ ) purchased from Merck KGaA (Darmstadt, Germany). Purification of synthesized compounds was performed on an Intelli Flash 310 Chromatograph from Varian Medical Systems (Darmstadt, Germany) using SF25-80

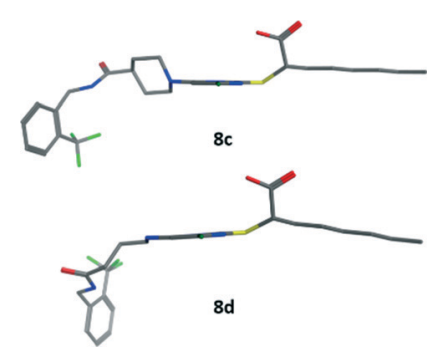

Fig. 3 Low-energy conformation of compounds $8 \mathrm{c}$ and $8 \mathrm{~d}$, calculated by quantum mechanics energy minimization software implemented in MOE2015.10 (Chemical Computing Group, Montreal, Canada). $\mathrm{g}$ and SF25-60 g columns, both loaded with silica gel (particle size $50 \mu \mathrm{m}$ ) and also purchased from Varian Medical Systems (Darmstadt, Germany). ${ }^{1} \mathrm{H}(250 / 400 \mathrm{MHz})$ and ${ }^{13} \mathrm{C}(64 \mathrm{MHz})$ were recorded on DPX250 and AV400 nuclear magnetic resonance spectrometers from Bruker (Karlsruhe, Germany). All spectra were analyzed with TopSpin software (Bruker, Karlsruhe, Germany). Tetramethylsilane was used as internal standard. DMSO- $d_{6}$ and methanol- $d_{4}$ were used as solvents. HPLC and mass analyses were performed on a LCMS 2020 from Shimadzu (Duisburg, Germany), under the use of a MultoHigh $100 \mathrm{RP} 18,3 \mu, 100 \times 2 \mathrm{~mm}$ column from CS ChromatographyService GmbH (Langerwehe, Germany) with an acetonitrile/ water gradient from $20-75 \%$. Electron spray ionization spectra were recorded in positive $(+)$ as well as negative $(-)$ mode and UV was measured at two wavelengths $(\lambda=254$ and $280 \mathrm{~nm})$. High resolution mass spectroscopy was performed by a Thermo Scientific MALDI LTQ ORBITRAP XL. All final compounds had a purity $\geq 95 \%$ as determined by HPLC.

Murine embryonic 3T3-L1 fibroblasts were purchased from the American Type Culture Collection (ATCC, Manassas, VA, USA). COS-7 african green monkey kidney cells originated from Deutsche Sammlung für Mikroorgansimen und Zellkulturen (DSMZ, Braunschweig, Germany).

General procedure for preparation of compounds $1 \mathrm{a}-\mathrm{b}$, using the example of ethyl 2-(4-cyanophenoxy)propanoate (1a). $5 \mathrm{~g}(25.2 \mathrm{mmol})$ 4-hydroxybenzonitrile, $20.9 \mathrm{~g}$ (151 mmol) potassium carbonate, $2.1 \mathrm{~g}(12.6 \mathrm{mmol})$ potassium iodide and $3.3 \mathrm{ml}(25.2 \mathrm{mmol})$ ethyl 2-bromopropanoate were refluxed in $50 \mathrm{ml}$ acetone for $72 \mathrm{~h}$. After cooling to room temperature, the mixture was filtered and the solvent was removed under reduced pressure. The crude residue was dissolved in $30 \mathrm{ml}$ ethyl acetate, washed three times with $2 \mathrm{M}$ sodium hydroxide solution and twice with $30 \mathrm{ml}$ brine. The organic layer was dried over magnesium sulfate and the solvent was removed under reduced pressure. After purification by flash chromatography, using pure dichloromethane, compound 1a remained as a white powder $(2.2 \mathrm{~g}, 40 \%) ;{ }^{1} \mathrm{H}$ NMR $\left(250 \mathrm{MHz}\right.$, DMSO- $\left.d_{6}\right)$ : $\delta=7.75(\mathrm{~d}, J=8.9 \mathrm{~Hz}, 2 \mathrm{H}), 7.03(\mathrm{~d}, J=8.8 \mathrm{~Hz}, 2 \mathrm{H}), 5.13(\mathrm{q}, J=$ $7.2 \mathrm{~Hz}, 1 \mathrm{H}), 4.13(\mathrm{q}, J=8.01 \mathrm{~Hz}, 2 \mathrm{H}), 1.52(\mathrm{~d}, J=6.9 \mathrm{~Hz}, 3 \mathrm{H})$, $1.15 \mathrm{ppm}(\mathrm{t}, J=7.3 \mathrm{~Hz}, 3 \mathrm{H})$; MS-ESI+: $\mathrm{m} / z 242[\mathrm{M}+\mathrm{Na}]^{+}$.

Ethyl 2-(4-(cyanomethyl)phenoxy)propanoate (1b). Remained as a white powder $(2 \mathrm{~g}, 38 \%) ;{ }^{1} \mathrm{H}$ NMR $\left(250 \mathrm{MHz}\right.$, DMSO- $\left.d_{6}\right)$ : $\delta=7.29-6.88(\mathrm{~m}, 4 \mathrm{H}), 4.69(\mathrm{q}, J=5.7 \mathrm{~Hz}, 1 \mathrm{H}), 4.15(\mathrm{q}, J=6 \mathrm{~Hz}$, $2 \mathrm{H}), 3.94(\mathrm{~s}, 2 \mathrm{H}), 1.51(\mathrm{~d}, J=6.8 \mathrm{~Hz}, 3 \mathrm{H}), 1.18 \mathrm{ppm}(\mathrm{t}, J=6.8$ $\mathrm{Hz}, 3 \mathrm{H})$; MS-ESI+: $m / z 256[\mathrm{M}+\mathrm{Na}]^{+}$.

General procedure for preparation of compounds $2 a-b$, using the example of ethyl 2-(4-(aminomethyl)phenoxy)propanoate (2a). $1 \mathrm{~g}$ (4.6 mmol) ethyl 2-(4-cyanophenoxy)propanoate (1a), $0.1 \mathrm{~g}(0.21 \mathrm{mmol})$ palladium on carbon $10 \%$ and $0.78 \mathrm{ml}(13.68 \mathrm{mmol})$ glacial acetic acid were stirred in $10 \mathrm{ml}$ dry ethanol under argon atmosphere for $24 \mathrm{~h}$. The catalyst was segregated over Celite ${ }^{\circledR}$ and the solvent was removed under reduced pressure. The crude product was dissolved in $25 \mathrm{ml}$ of $2 \mathrm{M}$ hydrochloric acid and washed with $20 \mathrm{ml}$ cyclohexane. To the aqueous solution was cooled to 5 ${ }^{\circ} \mathrm{C}$ and by adding $1 \mathrm{M}$ potassium hydroxide solution the $\mathrm{pH}$ 
Table 1 In vitro activity values of dual SEH/PPAR modulators

\begin{tabular}{|c|c|c|c|c|}
\hline Compd. & $\mathrm{IC}_{50} \mathrm{sEH}[\mu \mathrm{M}]$ & $\mathrm{EC}_{50} \operatorname{PPAR} \alpha[\mu \mathrm{M}]\left(E_{\max }-\%\right)$ & $\mathrm{EC}_{50} \operatorname{PPAR} \delta[\mu \mathrm{M}]\left(E_{\max }-\%\right)$ & $\mathrm{EC}_{50} \operatorname{PPAR} \gamma[\mu \mathrm{M}]\left(E_{\max }-\%\right)$ \\
\hline CIU & $0.14 \pm 0.02$ & ia. & ia. & (a10 $\mu \mathrm{M}(22 \%)$ \\
\hline MD78 & ia. & $0.067 \pm 0.004 \mu \mathrm{M}(95 \%)$ & ia. & $0.69 \pm 0.02 \mu \mathrm{M}(126 \%)$ \\
\hline $3 \mathbf{a}$ & $0.064 \pm 0.002$ & ia. & ia. & ia. \\
\hline $3 \mathbf{b}$ & $0.027 \pm 0.008$ & ia. & ia. & ia. \\
\hline $3 \mathrm{c}$ & $0.85 \pm 0.003$ & ia. & ia. & ia. \\
\hline $5 \mathbf{b}$ & $0.023 \pm 0.008$ & ia. & ia. & ia. \\
\hline $5 \mathrm{c}$ & $0.075 \pm 0.007$ & ia. & ia. & ia. \\
\hline 5d & $0.027 \pm 0.004$ & ia. & ia. & ia. \\
\hline $8 a$ & $2.5 \pm 0.5$ & ia. & ia. & ia. \\
\hline $8 \mathbf{b}$ & $1.3 \pm 0.3$ & ia. & ia. & ia. \\
\hline $8 \mathrm{c}$ & $0.3 \pm 0.04$ & (@10 $\mu \mathrm{M}(65 \%)$ & ia. & ia. \\
\hline
\end{tabular}

was adjusted to a basic level. The aqueous layer was extracted with $25 \mathrm{ml}$ ethyl acetate. The organic layer was dried over magnesium sulfate and the solvent was removed under reduced pressure. Without further purification, 2a remained as clear oil (0.69 g, 68\%); ${ }^{1} \mathrm{H}$ NMR (250 MHz, DMSO- $\left.d_{6}\right): \delta=$ 7.21-6.6 (m, 4H), 4.77 (q, $J=6.8 \mathrm{~Hz}, 1 \mathrm{H}), 4.12(\mathrm{q}, J=6.7 \mathrm{~Hz}$, 2H), $2.65(\mathrm{~s}, 2 \mathrm{H}), 1.5(\mathrm{~d}, J=6.8 \mathrm{~Hz}, 3 \mathrm{H}), 1.17 \mathrm{ppm}(\mathrm{t}, J=6.9$ $\mathrm{Hz}, 3 \mathrm{H})$; MS-ESI+: $m / z 246[\mathrm{M}+\mathrm{Na}]^{+}$.

Ethyl 2-(4-(aminoethyl)phenoxy)propanoat (2b). Remained as clear oil (0.67 g, 66\%); ${ }^{1} \mathrm{H}$ NMR (250 MHz, DMSO- $\left.d_{6}\right): \delta=$ 7.13-6.75 (m, 4H,), 4.87 (q, $J=6.9 \mathrm{~Hz}, 1 \mathrm{H}), 4.14(\mathrm{q}, J=7 \mathrm{~Hz}$, $2 \mathrm{H}), 2.7(\mathrm{t}, J=6.1,2 \mathrm{H}), 2.6(\mathrm{t}, J=6.5,2 \mathrm{H}), 1.49(\mathrm{~d}, J=6.8 \mathrm{~Hz}$, $3 \mathrm{H}), 1.18 \mathrm{ppm}(\mathrm{t}, J=6.8 \mathrm{~Hz})$; MS-ESI ${ }^{+}: \mathrm{m} / \mathrm{z} 260[\mathrm{M}+\mathrm{Na}]^{+}$.

General procedure for preparation of compounds $3 a-b$, using the example of ethyl 2-(4-((3-(4-chlorophenyl)ureido)methyl)phenoxy)propanoate (3a). $0.5 \mathrm{~g}$ (2.24 $\mathrm{mmol})$ ethyl 2-(4-(aminomethyl)phenoxy)propanoate (2a), $0.59 \mathrm{ml}$ (3.36 mmol) $N, N$-diisopropylethylamine and $0.29 \mathrm{ml}(2.24 \mathrm{mmol})$ 4-chlorophenyl isocyanate were stirred in $5 \mathrm{ml}$ dry dichloromethane under argon for $24 \mathrm{~h}$. Then, the reaction mixture was diluted with further $10 \mathrm{ml}$ dichloromethane and washed with $1 \mathrm{M}$ hydrochloric acid. The organic layer was dried over magnesium sulfate and the solvent was removed under reduced pressure. The crude product was purified by flash chromatography with hexane/ethyl acetate 3:1. Compound 3a remained as a white powder $(0.17 \mathrm{~g}, 20 \%) ;{ }^{1} \mathrm{H}$ NMR $(250 \mathrm{MHz}$, DMSO- $\left.d_{6}\right): \delta=8.7(\mathrm{~s}, 1 \mathrm{H}), 7.5-6.6(\mathrm{~m}, 8 \mathrm{H}), 5.78(\mathrm{t}, J=6.3 \mathrm{~Hz}$, $1 \mathrm{H}), 4.92(\mathrm{q}, J=6.7 \mathrm{~Hz}, 1 \mathrm{H}), 4.22(\mathrm{~d}, J=5.7 \mathrm{~Hz}, 2 \mathrm{H}), 4.14(\mathrm{q}, J=$ $6.8 \mathrm{~Hz}, 2 \mathrm{H}), 1.5(\mathrm{~d}, J=6.7 \mathrm{~Hz}, 3 \mathrm{H}), 1.18 \mathrm{ppm}(\mathrm{t}, J=7.6 \mathrm{~Hz}, 3 \mathrm{H})$; ${ }^{13} \mathrm{C}$ NMR (75.4 MHz, DMSO- $d_{6}$ ): $172,159.1,154.1,134.9,132.3$, 129.4, 129.3, 129.1, 128.6, 128.4, 122, 119.1, 114.3, 114.6, 79, 61.3, 50.9, 17.2, $12.3 \mathrm{ppm}$; HRMS-MALDI $\mathrm{m} / z[\mathrm{M}+\mathrm{H}]^{+}$calcd for $\mathrm{C}_{19} \mathrm{H}_{21} \mathrm{ClN}_{2} \mathrm{O}_{4}$ : 376.1193, found 376.1192.

Ethyl 2-(4-((3-(4-chlorophenyl)ureido)ethyl)phenoxy)propanoate (3b). Remained as a white powder (0.15 g, 18\%); ${ }^{1} \mathrm{H}$ NMR (250 MHz, DMSO- $\left.d_{6}\right): \delta=8.62(\mathrm{~s}, 1 \mathrm{H}), 7.44-6.8(\mathrm{~m}, 8 \mathrm{H}), 6.13(\mathrm{t}$, $J=5.3 \mathrm{~Hz}, 1 \mathrm{H}), 4.9(\mathrm{q}, J=6.7 \mathrm{~Hz}, 1 \mathrm{H}), 4.15(\mathrm{q}, J=8 \mathrm{~Hz}, 2 \mathrm{H})$, $2.68(\mathrm{t}, J=8.1 \mathrm{~Hz}, 2 \mathrm{H}), 1.5(\mathrm{~d}, J=6.1 \mathrm{~Hz}, 3 \mathrm{H}), 1.18 \mathrm{ppm}(\mathrm{t}, J=$ $6.6 \mathrm{~Hz}, 3 \mathrm{H}) ;{ }^{13} \mathrm{C}$ NMR (75.4 MHz, DMSO- $d_{6}$ ): $173,162.1,149.1$,
136.9, 134.3, 130.4, 129.7, 129.2, 128.9, 128.6, 120, 119.5, 114.9, 114, 80, 61.4, 48.5, 36.7, 18.2, $11.3 \mathrm{ppm}$; HRMS-MALDI $\mathrm{m} / \mathrm{z}$ $[\mathrm{M}+\mathrm{H}]^{+}$calcd for $\mathrm{C}_{20} \mathrm{H}_{23} \mathrm{ClN}_{2} \mathrm{O}_{4}: 390.1351$, found 390.1352.

General procedure for synthesis of compounds $3 c-d, 5 c, 5 d$ using the example of 2-(4-((3-(4-chlorophenyl)ureido)methyl)phenoxy)propionic acid (3c). $100 \mathrm{mg}$ (0.27 mmol) ethyl 2-(4-((3-(4-chlorophenyl)ureido)methyl)phenoxy)propanoate (3a) and $74 \mathrm{mg}(1.33 \mathrm{mmol})$ potassium hydroxide were dissolved in $2 \mathrm{ml}$ of a solvent mixture containing tetrahydrofuran, water and methanol in a ratio $1 / 2 / 1$ and stirred under microwave irradiation in a Initiator Microwave Synthesizer from Biotage (Uppsala, Sweden) at $70{ }^{\circ} \mathrm{C}$ for 30 minutes. The organic solvents were removed under reduced pressure. With the addition of a few drops $12 \mathrm{M}$ hydrochloric acid 3c precipitated in the aqueous phase. After filtration the product was dried by lyophilization and $3 \mathrm{c}$ remained as white powder (42 $\mathrm{mg}, 45 \%) ;{ }^{1} \mathrm{H}$ NMR (250 MHz, DMSO- $\left.d_{6}\right): \delta=8.65$ (s, $\left.1 \mathrm{H}\right)$, 7.45-6.8 (m, 8H), $6.58(\mathrm{t}, J=5.75 \mathrm{~Hz}, 1 \mathrm{H}), 4.8(\mathrm{q}, J=6.5 \mathrm{~Hz}$, $1 \mathrm{H}), 4.21$ (d, $J=6.1 \mathrm{~Hz}, 2 \mathrm{H}), 1.49 \mathrm{ppm}(\mathrm{d}, J=7.2 \mathrm{~Hz}, 3 \mathrm{H}) ;{ }^{13} \mathrm{C}$ NMR (75.4 MHz, DMSO- $d_{6}$ ): 178, 159.1, 147.1, 135.9, 133.3, 131.4, 129.9, 129.7, 129, 128.7, 119, 118.1, 114.5, 113, 76, 49.5, $19.2 \mathrm{ppm}$, HRMS-MALDI $m / z[\mathrm{M}+\mathrm{H}]^{+}$calcd for $\mathrm{C}_{17} \mathrm{H}_{17} \mathrm{ClN}_{2} \mathrm{O}_{4}$ : 348.0885 , found 348.0883 .

2-(4-((3-(4-Chlorophenyl)ureido)ethyl)phenoxy)propionic acid (3d). Remained as a white powder (39 mg, 42\%); ${ }^{1} \mathrm{H}$ NMR (250 MHz, DMSO- $\left.d_{6}\right): \delta=8.63(\mathrm{~s}, 1 \mathrm{H}), 7.44-6.79(\mathrm{~m}$, $8 \mathrm{H}), 6.14(\mathrm{t}, J=6 \mathrm{~Hz}, 1 \mathrm{H}), 4.78(\mathrm{q}, J=6.5 \mathrm{~Hz}, 1 \mathrm{H}), 3.2(\mathrm{q}, J=$ $6.9 \mathrm{~Hz}, 2 \mathrm{H}), 1.49 \mathrm{ppm}(\mathrm{d}, J=6.5 \mathrm{~Hz}, 3 \mathrm{H}) ;{ }^{13} \mathrm{C}$ NMR $(75.4 \mathrm{MHz}$, DMSO- $\left.d_{6}\right): 180,159.1,148.1,137.2$, 135.3, 129.9, 129.6, 129.2, 128.9, 128.5, 118, 117.5, 114.8, 114.1, 79.1, 49.5, 36.5, 18.1 ppm; HRMS-MALDI $\mathrm{m} / \mathrm{z}[\mathrm{M}+\mathrm{H}]^{+}$calcd for $\mathrm{C}_{18} \mathrm{H}_{19} \mathrm{ClN}_{2} \mathrm{O}_{4}$ : 362.1032, found 362.1031.

General procedure for amide synthesis of compounds $4,6 \mathrm{a}$ and $6 \mathrm{~b}$, described with the synthesis of 1-(4-formylphenyl)- $\mathrm{N}$ (2-(trifluoromethyl)benzyl)piperidine-4-carboxamide (4). $1 \mathrm{~g}$ (4.29 mmol) 1-(4-formylphenyl)piperidine-4-carbonic acid, 0.66 $\mathrm{ml}(4.72 \mathrm{mmol})$ triethylamine and $0.61 \mathrm{ml}(4.72 \mathrm{mmol})$ isobutyl chloroformiate were stirred in $40 \mathrm{ml}$ dry chloroform at $0{ }^{\circ} \mathrm{C}$ under argon atmosphere for $1 \mathrm{~h}$. Subsequently $0.66 \mathrm{ml}$ 
(4.29 mmol) 2-(trifluoromethyl)benzylamine were added and the mixture was further stirred at room temperature for $12 \mathrm{~h}$. The reaction mixture was washed three times with $20 \mathrm{ml}$ of 2 $\mathrm{M}$ hydrochloric acid, three times with $20 \mathrm{ml}$ of $2 \mathrm{M}$ sodium hydroxide solution and two times with $20 \mathrm{ml}$ brine. The organic layer was dried over magnesium sulfate and the solvent was removed under reduced pressure. The product was recrystallized from ethyl acetate/hexane. Compound 4 remained as a white powder $(0.9 \mathrm{~g}, 54 \%) ;{ }^{1} \mathrm{H}$ NMR $\left(250 \mathrm{MHz}, \mathrm{DMSO}-d_{6}\right): \delta=9.8$ (s, $1 \mathrm{H}), 8.56(\mathrm{t}, J=6.4 \mathrm{~Hz}, 1 \mathrm{H}), 7.82-7.12(\mathrm{~m}, 8 \mathrm{H}), 4.53(\mathrm{~d}, J=6.1$ $\mathrm{Hz}, 2 \mathrm{H}), 3.93-2.9$ (m, 4H), 2.5 (m, 1H), 1.88-1.74 ppm (m, 4H); MS-ESI ${ }^{-}: m / z 425[\mathrm{M}+\mathrm{Cl}]^{-}$.

Preparation of $(E)-2-(4-(4-((2-$ trifluoromethyl)benzyl)carbamoyl)piperidine-1-yl)benzylidene)-alpha-ethylcinnamate (5a). $200 \mathrm{mg}$ (5.12 mmol) sodium hydride $63 \%$ and $9.1 \mathrm{ml}$ (3.84 mmol) triethyl 2-phosphonobutyrate were stirred in $5 \mathrm{ml}$ dry tetrahydrofuran at $0{ }^{\circ} \mathrm{C}$ under argon atmosphere for 30 minutes. Then, $1 \mathrm{~g}(2.56 \mathrm{mmol})$ 1-(4-formylphenyl)- $N$-(2-(trifluoromethyl)benzyl)piperidine-4carboxamide (4) was dissolved in $10 \mathrm{ml}$ dry tetrahydrofuran and added to the reaction mixture. After $2 \mathrm{~h}$ the reaction was stopped with the addition of $25 \mathrm{ml}$ water. The mixture was diluted with 10 $\mathrm{ml}$ ethyl acetate and washed three times with brine. The organic layer was dried over magnesium sulfate and the solvent was removed under reduced pressure. The product was recrystallized from ethyl acetate/hexane. Compound $5 \mathrm{a}$ remained as a white powder $(901 \mathrm{mg}$, $72 \%) ;{ }^{1} \mathrm{H}$ NMR (250 MHz, DMSO- $d_{6}$ ): $\delta=8.5$ (t, $\left.J=6 \mathrm{~Hz}, 1 \mathrm{H}\right), 7.75-$ 7.05 (m, 9H), 4.47 (d, $J=7.4 \mathrm{~Hz}, 2 \mathrm{H}), 4.2$ (q, $J=6.3 \mathrm{~Hz}, 2 \mathrm{H}), 3.92-2.8$ (m, 4H), 2.9 (q, $J=12 \mathrm{~Hz}, 2 \mathrm{H}), 2.5(\mathrm{~m}, 1 \mathrm{H}), 1.92-1.68(\mathrm{~m}, 4 \mathrm{H}), 1.29$ $(\mathrm{t}, J=7.3 \mathrm{~Hz}, 3 \mathrm{H}), 1.14 \mathrm{ppm}(\mathrm{t}, J=8.3 \mathrm{~Hz}, 3 \mathrm{H}) ;{ }^{13} \mathrm{C} \mathrm{NMR}(75.4 \mathrm{MHz}$, DMSO- $\left.d_{6}\right)$ : $178.9,165,142.9,139.5,135,132.1,132,128,129.1,127.9$, $127.8,126.5,125.2,124.5,113,113.2,112,60,51.6,51,39.3,25.9$, 25.8, 19.6, 13.9, 11.1; HRMS-MALDI $\mathrm{m} / z[\mathrm{M}+\mathrm{H}]^{+}$calcd for $\mathrm{C}_{27} \mathrm{H}_{31} \mathrm{~F}_{3} \mathrm{~N}_{2} \mathrm{O}_{3}$ : 488.2295, found 488.2295.

(E)-2-(4-(4-((2-(Trifluoromethyl)benzyl)carbamoyl)piperidine1-yl)benzylidene)-alpha-ethylcinnamic acid (5d). 5d remained as a white powder (57 mg, 60\%); ${ }^{1} \mathrm{H}$ NMR (250 MHz, DMSO$\left.d_{6}\right): \delta=8.49(\mathrm{t}, J=5.7 \mathrm{~Hz}, 1 \mathrm{H}), 7.72-7.1(\mathrm{~m}, 9 \mathrm{H}), 4.44(\mathrm{~d}, J=5.4$ $\mathrm{Hz}, 2 \mathrm{H}), 3.93-2.86(\mathrm{~m}, 4 \mathrm{H}), 2.8$ (q, $J=9 \mathrm{~Hz}, 2 \mathrm{H}), 2.49(\mathrm{~m}, 1 \mathrm{H})$, 1.9-1.7 (m, 4H), $1.09 \mathrm{ppm}(\mathrm{t}, J=7.1 \mathrm{~Hz}, 3 \mathrm{H}) ;{ }^{13} \mathrm{C}$ NMR (75.4 MHz, DMSO- $\left.d_{6}\right): 179,170.1,142.7,136.5,134,132.5,132.1$, 127, 129.5, 127.1, 126.9, 126, 125.1, 124.4, 113.1, 113, 111, 51.7, 52, 38.3, 26.1, 25.7, 19.3, 12.5; HRMS-MALDI $m / z[\mathrm{M}+\mathrm{H}]^{+}$calcd for $\mathrm{C}_{25} \mathrm{H}_{27} \mathrm{~F}_{3} \mathrm{~N}_{2} \mathrm{O}_{3}$ : 460.2200, found 460.2201.

Preparation of ethyl 2-(4-(4-((2-(trifluoromethyl)benzyl)carbamoyl)piperidine-1-yl)benzyl)butanoate (5b). $200 \mathrm{mg}$ (0.41 mmol) (E)-2-(4-(4-((2-(trifluoromethyl)benzyl)carbamoyl)piperidine-1-yl)benzylidene)-alpha-ethylcinnamate (5a) and 100 mg magnesium chips were stirred with $4 \mathrm{ml}$ dry methanol under argon atmosphere. After $12 \mathrm{~h}$ the reaction was stopped by addition of $4 \mathrm{ml} 2 \mathrm{M}$ hydrochloric acid. The product was extracted with $10 \mathrm{ml}$ ethyl acetate. The organic layer was dried over magnesium sulfate and the solvent was removed under reduced pressure. For purification a flash chromatography was used with ethyl acetate and hexane $(1: 1)$ as mobile phase. $5 \mathbf{d}$ remained as white powder $(161 \mathrm{mg}, 80 \%) ;{ }^{1} \mathrm{H}$ NMR $(250 \mathrm{MHz}$, DMSO- $\left.d_{6}\right): \delta=8.56(\mathrm{t}, J=5.6 \mathrm{~Hz}, 1 \mathrm{H}), 7.81-7.04(\mathrm{~m}, 8 \mathrm{H}), 4.52$ (d, $J=6.2 \mathrm{~Hz}, 2 \mathrm{H}), 4.07$ (q, $J=7.2 \mathrm{~Hz}, 2 \mathrm{H}), 3.92-2.65(\mathrm{~m}, 4 \mathrm{H})$, $2.74(\mathrm{q}, J=8 \mathrm{~Hz}), 2.4(\mathrm{~m}, 1 \mathrm{H}), 2.3-2.29(\mathrm{~m}, 1 \mathrm{H}), 1.98-1.72(\mathrm{~m}$, $4 \mathrm{H}), 1.23(\mathrm{t}, J=6.3 \mathrm{~Hz}, 3 \mathrm{H}), 1.14 \mathrm{ppm}(\mathrm{t}, J=7.9 \mathrm{~Hz}, 3 \mathrm{H}) ;{ }^{13} \mathrm{C}-$ NMR (75.4 MHz, DMSO- $d_{6}$ ): 178.1, 174.2, 142.8, 138.5, 131, 129.2, 129.1, 128, 127.9, 126.4, 125.1, 124.1, 113, 112.9, 112, 59.8, 51.7, 51, 48, 39.1, 35, 26.1, 25.8, 19.6, 14.1, 13.1; HRMS-FAB m/z $[\mathrm{M}+\mathrm{H}]^{+}$calcd for $\mathrm{C}_{27} \mathrm{H}_{32} \mathrm{~F}_{3} \mathrm{~N}_{2} \mathrm{O}_{3}:$ 490.2442, found 490.2441.

2-(4-(4-((2-(Trifluoromethyl)benzyl)carbamoyl)piperidine-1yl)benzyl)butanoic acid (5c). 5c remained as a white powder (58 mg, 61\%); ${ }^{1} \mathrm{H}$ NMR $\left(250 \mathrm{~Hz}\right.$, DMSO- $\left.d_{6}\right): \delta=6.64(\mathrm{t}, J=6.1$ $\mathrm{Hz}, 1 \mathrm{H}), 7.81-7.29(\mathrm{~m}, 8 \mathrm{H}), 4.53$ (d, $J=6.1 \mathrm{~Hz}, 2 \mathrm{H}), 3.77-2.7$ $(\mathrm{m}, 4 \mathrm{H}), 2.6(\mathrm{q}, J=7 \mathrm{~Hz}, 2 \mathrm{H}), 2.5(\mathrm{~m}, 1 \mathrm{H}), 2.31-2.28(\mathrm{~m}, 1 \mathrm{H})$, 1.98-2.11 (m, 4H), $0.94 \mathrm{ppm}(\mathrm{t}, J=7.4 \mathrm{~Hz}, 3 \mathrm{H}) ;{ }^{13} \mathrm{C}-\mathrm{NMR}(75.4$ MHz, DMSO- $\left.d_{6}\right)$ : 179.5, 178.1, 142.1, 137.5, 132, 129, 128.9, 128, 127.8, 125.9, 125.1, 124.6, 114, 113.9, 112, 51.9, 50.1, 49, 39.4, 34, 26.1, 25.7, 18.1, 12.1; HRMS-FAB $\mathrm{m} / z[\mathrm{M}+\mathrm{H}]^{+}$calcd for $\mathrm{C}_{25} \mathrm{H}_{29} \mathrm{~F}_{3} \mathrm{~N}_{2} \mathrm{O}_{3}$ : 462.2133, found 462.2131.

tert-Butyl 4-((2-(trifluoromethyl)benzyl)carbamoyl)piperidine1-carboxylate (6a). 6a remained as an unclear oil (1.146 g, 68\%); ${ }^{1} \mathrm{H}$ NMR (250 MHz, DMSO- $\left.d_{6}\right): \delta=8.49(\mathrm{t}, J=5.4 \mathrm{~Hz}, 1 \mathrm{H}), 7.78-$ $7.49(\mathrm{~m}, 4 \mathrm{H}), 4.49(\mathrm{~d}, J=5.4 \mathrm{~Hz}, 2 \mathrm{H}), 4.06-3.96(\mathrm{~m}, 2 \mathrm{H}), 2.88-$ $2.72(\mathrm{~m}, 2 \mathrm{H}), 2.51-2.44(\mathrm{~m}, 1 \mathrm{H}), 1.83-1.73$ (m, 2H), 1.51-1.5 (m, 2H), 1.45 ppm (s, 9H). MS-ESI ${ }^{-}: m / z 410\left[\mathrm{M}+\mathrm{Cl}^{-}\right]$.

tert-Butyl (4-oxo-4-((2-(trifluoromethyl)benzyl)amino)butyl)carbamate (6b). 6b remained as an unclear oil (0.975 g, 55\%); ${ }^{1} \mathrm{H}$ NMR (250 MHz, DMSO- $\left.d_{6}\right): \delta=8.45(\mathrm{t}, J=5.9 \mathrm{~Hz}, 1 \mathrm{H}), 7.79-$ $7.5(\mathrm{~m}, 4 \mathrm{H}), 6.88(\mathrm{t}, J=9.1 \mathrm{~Hz}, 1 \mathrm{H}), 4.49$ (d, $J=6 \mathrm{~Hz}, 2 \mathrm{H}), 2.98$ (q, $J=6.6 \mathrm{~Hz}, 2 \mathrm{H}), 2.24(\mathrm{t}, J=7.2 \mathrm{~Hz}, 2 \mathrm{H}), 1.7(\mathrm{q}, J=7.3 \mathrm{~Hz}$, $2 \mathrm{H}), 1.44 \mathrm{ppm}(\mathrm{s}, 9 \mathrm{H}) . \mathrm{MS}_{-\mathrm{ESI}}^{+}: \mathrm{m} / z 383[\mathrm{M}+\mathrm{Na}]^{+}$.

General procedure of Boc-protecting group cleavage for compounds $7 \mathrm{a}$ and $7 \mathrm{~b}$, described with the synthesis of $\mathrm{N}$-(2(trifluoromethyl)benzyl)piperidine-4-carboxamide (7a). $230 \mathrm{mg}$ (0.6 mmol) tert-butyl 4-((2-(trifluoromethyl)benzyl)carbamoyl)piperidine-1-carboxylate (6a) and $0.92 \mathrm{ml}$ (11.9 $\mathrm{mmol})$ trifluoroacetic acid were stirred in $4 \mathrm{ml}$ dichloromethane for 60 minutes. The organic solvent was removed under reduced pressure and the crude residue was dissolved in $10 \mathrm{ml}$ of $2 \mathrm{M}$ hydrochloric acid. The acidic solution was washed twice with $10 \mathrm{ml}$ ethyl acetate. By the addition of $8 \mathrm{M}$ sodium hydroxide solution the $\mathrm{pH}$ was adjusted to a basic level and the product was extracted with ethyl acetate $(3 \times 15 \mathrm{~mL})$. The organic layer was dried over magnesium sulfate and the solvent was removed under reduced pressure. Without further purification, 7a remained as a colorless oil $(0.13 \mathrm{~g}, 76 \%) ;{ }^{1} \mathrm{H}$ NMR $\left(250 \mathrm{MHz}\right.$, DMSO- $\left.d_{6}\right): \delta=$ $8.42(\mathrm{t}, J=5.6 \mathrm{~Hz}, 1 \mathrm{H}), 7.79-7.48(\mathrm{~m}, 4 \mathrm{H}), 4.48(\mathrm{~d}, J=6.2 \mathrm{~Hz}, 2 \mathrm{H})$, 3.08-2.99 (m, 2H), 2.61-2.5 (m, 2H), 2.44-2.33 (m, 1H), 1.78-1.68 ppm (m, 2H), 1.46-1.6 (m, 2H). MS-ESI ${ }^{+}: m / z 287\left[\mathrm{M}+\mathrm{H}^{+}\right]$.

4-Amino- $\mathrm{N}$-(2-(trifluoromethyl)benzyl)butanamide (7b). $7 \mathrm{~b}$ remained as a colorless oil $(71 \mathrm{mg}, 50 \%) ;{ }^{1} \mathrm{H}$ NMR $(250 \mathrm{MHz}$, DMSO- $\left.d_{6}\right): \delta=8.4(\mathrm{t}, J=5.4 \mathrm{~Hz}, 1 \mathrm{H}), 7.74-7.45(\mathrm{~m}, 4 \mathrm{H}), 4.44$ $(\mathrm{d}, J=5.6 \mathrm{~Hz}, 2 \mathrm{H}), 2.66(\mathrm{t}, J=7.4 \mathrm{~Hz}, 2 \mathrm{H}), 2.23(\mathrm{t}, J=7.1 \mathrm{~Hz}$, 2H), $1.62 \mathrm{ppm}(\mathrm{q}, J=7.1 \mathrm{~Hz}, 2 \mathrm{H}) . \mathrm{MS}^{-E_{S I}^{+}}{ }^{\prime} \mathrm{m} / z 261\left[\mathrm{M}+\mathrm{H}^{+}\right]$.

General procedure of compounds $8 \mathrm{a}$ and $8 \mathrm{~b}$ is described by the synthesis of ethyl 2-((4-chloro-6-(4-((2-(trifluoromethyl)benzyl)carbamoyl)piperidine-1-yl)pyrimidine-2-yl)thio)octanoate (8a). $100 \mathrm{mg}$ ethyl 2-((4,6-dichloropyrimidine-2-yl)thio)octanoate 
were dissolved in $2 \mathrm{ml}$ dry acetonitrile under argon atmosphere. A mixture of $0.04 \mathrm{ml}(0.285 \mathrm{mmol})$ triethylamine and $81 \mathrm{mg}$ (0.285 mmol) $\mathrm{N}$-(2-(trifluoromethyl)benzyl)piperidine-4-carboxamide (7a) were added slowly and the mixture was stirred for 12 h. With the addition of water, the product precipitated, was filtered off and dried by lyophilization. Without further purification, 8a remained as a white powder $(0.116 \mathrm{~g}, 68 \%) ;{ }^{1} \mathrm{H}$ NMR (250 MHz, methanol- $d_{4}: \delta=7.62-7.31(\mathrm{~m}, 4 \mathrm{H}), 6.44(\mathrm{~s}, 1 \mathrm{H}), 4.47$ (s, 2H), $4.22(\mathrm{t}, J=7.2 \mathrm{~Hz}, 1 \mathrm{H}), 4.07$ (q, $J=7.3 \mathrm{~Hz}, 2 \mathrm{H}), 2.99-2.87$ (m, 2H), 2.62-2.47 (m, 1H), 1.9-1.48 (m, 6H), 1.47-1.11 (m, 13H), $0.8 \mathrm{ppm}(\mathrm{t}, J=7.6 \mathrm{~Hz}, 3 \mathrm{H}) ;{ }^{13} \mathrm{C}$ NMR (75.4 MHz, methanol- $\left.d_{4}\right)$ : 178.8, 174.6, 171.2, 167.2, 160.6, 139.3, 131.7, 129.2, 127.4, 126.7, 125.1, 113.1, 102.8, 60.1, 52, 51.2, 44.7, 39.1, 35.2, 30.1, 29.8, 27.5, 25.7, 25.8, 32.6, 23.1, 14, 13.6; HRMS-MALDI $m / z[\mathrm{M}+\mathrm{H}]^{+}$ calcd for $\mathrm{C}_{27} \mathrm{H}_{35} \mathrm{ClF}_{3} \mathrm{~N}_{2} \mathrm{O}_{3}: 600.2154$, found 600.2153.

Ethyl 2-((4-chloro-6-((4-oxo-4-((2-(trifluoromethyl)benzyl)amino)butyl)amino)pyrimidin-2-yl)thio)octanoat (8b). $8 \mathrm{~b}$ remained as a white powder $(0.508 \mathrm{~g}, 50 \%) ;{ }^{1} \mathrm{H}$ NMR $\left(250 \mathrm{MHz}\right.$, methanol- $\left.d_{4}\right): \delta$ $=7.62-7.3(\mathrm{~m}, 4 \mathrm{H}), 6.07(\mathrm{~s}, 1 \mathrm{H}), 4.47(\mathrm{~s}, 2 \mathrm{H}), 4.29(\mathrm{t}, J=7.3 \mathrm{~Hz}$, $1 \mathrm{H}), 4.04(\mathrm{q}, J=7.1 \mathrm{~Hz}, 2 \mathrm{H}), 3.49-3.27(\mathrm{~m}, 2 \mathrm{H}), 2.27(\mathrm{t}, J=7.2 \mathrm{~Hz}$, $2 \mathrm{H}), 1.82(\mathrm{q}, J=7.8 \mathrm{~Hz}, 2 \mathrm{H}), 1.38-1.11(\mathrm{~m}, 13 \mathrm{H}), 0.79(\mathrm{t}, J=7.1 \mathrm{~Hz}$, $3 \mathrm{H}) \mathrm{ppm} ;{ }^{13} \mathrm{C}$ NMR $\left(75.4 \mathrm{MHz}\right.$, methanol- $\left.d_{4}\right): 178.9,175.6,170.2$, 166.2, 158.6, 137.3, 134.7, 129.2, 128.4, 128.7, 126.1, 114.1, 103.8, 58.1, 50.6, 43.7, 34.2, 32.6, 31.7, 29.1, 28.8, 27.5, 27.4, 23.1, 14.7, 12.6; HRMS-MALDI $m / z[\mathrm{M}+\mathrm{H}]^{+}$calcd for $\mathrm{C}_{25} \mathrm{H}_{33} \mathrm{ClF}_{3} \mathrm{~N}_{2} \mathrm{O}_{3}$ : 574.1997, found 574.1998.

2-((4-Chloro-6-(4-((2-(trifluoromethyl)benzyl)carbamoyl)piperidine-1-yl)pyrimidine-2-yl)thio)octanoic acid (8c). To a solution of $8 \mathrm{~b}(0.3 \mathrm{~g}, 0.5 \mathrm{mmol})$ in THF $(10 \mathrm{~mL})$ was added $\mathrm{LiOH} \cdot \mathrm{H}_{2} \mathrm{O}$ (0.06 g, $\left.1.5 \mathrm{mmol}\right)$ dissolved in water $(2 \mathrm{~mL})$. The reaction was stirred for $24 \mathrm{~h}$ at $45{ }^{\circ} \mathrm{C}$. The organic solvent was removed under reduced pressure, and the residue was diluted with $3 \mathrm{ml}$ of water. The aqueous layer was acidified by addition of few drops of $12 \mathrm{M} \mathrm{HCl}$ solution. The resulting precipitate was separated by filtration and without further purification, 8c remained as a white powder (70 $\mathrm{mg}, 57 \%$ ); ${ }^{1} \mathrm{H}$ NMR (250 MHz, methanol- $\left.d_{4}\right): \delta=7.63-7.31(\mathrm{~m}, 4 \mathrm{H}), 6.39$ $(\mathrm{s}, 1 \mathrm{H}), 4.47(\mathrm{~s}, 2 \mathrm{H}), 4.19(\mathrm{t}, J=7.2 \mathrm{~Hz}, 1 \mathrm{H}), 2.98-2.86(\mathrm{~m}$, 2H), 2.61-2.48 (m, 1H), 1.9-1.53 (m, 6H), 1.29-1.11 ppm (m, 13H). ${ }^{13} \mathrm{C}$ NMR (75.4 MHz, methanol- $d_{4}$ ): 179.5, 178.1, 171.1, 165.2 , 159.6, 137.3, 132.7, 128.2, 128.1, 126.6, 125, 112.1, 102.9, 48.1, 48, 43.7, 40, 33.2, 32.1, 31.6, 28.8, 27.1, 25.1, 25, 21.1, 12, 11.6; HRMS-FAB $m / z[\mathrm{M}+\mathrm{H}]^{+}$calcd for $\mathrm{C}_{25} \mathrm{H}_{31} \mathrm{ClF}_{3} \mathrm{~N}_{2} \mathrm{O}_{3}$ : 572.1850, found 572.1849.

2-((4-Chloro-6-((4-oxo-4-((2-(trifluoromethyl)benzyl)amino)butyl)amino)pyrimidine-2-yl)thio)octanoic acid (8d) was synthesized according to the procedure described with the synthesis of compound 8c. 8d remained as a white powder (31 $\mathrm{mg}, 33 \%) ;{ }^{1} \mathrm{H}$ NMR (250 MHz, methanol- $\left.d_{4}\right): \delta=7.62-7.32$ $(\mathrm{m}, 4 \mathrm{H}), 6.03(\mathrm{~s}, 1 \mathrm{H}), 4.47(\mathrm{~s}, 2 \mathrm{H}), 4.24(\mathrm{t}, J=7.3 \mathrm{~Hz}, 1 \mathrm{H})$, 3.51-3.33 (m, 2H), 2.25-2.35 (m, 2H), 1.96-1.73 (m, 2H), 1.45-1.12 ppm (m, 13H); ${ }^{13} \mathrm{C}$ NMR (75.4 MHz, methanol- $\left.d_{4}\right)$ : $181.1,177.9$, 171.2, 165.2, 157.6, 138.3, 133.7, 128.2, 128.1, 127.7, 126.9, 113.1, 101.8, 51.6, 42.7, 33.2, 31.6, 30, 29.5, 28.7, 27, 26.1, 21.1, 13.7; HRMS-MALDI $m / z[\mathrm{M}+\mathrm{H}]^{+}$calcd for $\mathrm{C}_{23} \mathrm{H}_{29} \mathrm{ClF}_{3} \mathrm{~N}_{2} \mathrm{O}_{3}$ : 546.1688, found 546.1683.

\section{PPAR activity assay}

Evaluation of PPAR activity was performed as published before. ${ }^{24}$ In brief, COS-7 cells were grown in DMEM high glucose, supplemented with 10\% fetal calf serum (FCS), $1 \%$ sodium pyruvate (SP) and 1\% penicillin/streptomycin (PS) at 37 ${ }^{\circ} \mathrm{C}$ and $5 \% \mathrm{CO}_{2}$. The day before transfection, COS-7 cells were seeded in 96-well plates with a density of 30000 cells per well. Transient transfection was carried out using Lipofectamine LTX reagent (Invitrogen, Carlsbad, CA, USA) according to the manufacturer's protocol with pFR-Luc (Stratagene), pRL-SV40 (Promega) and the Gal4-fusion receptor plasmids (pFA-CMV-hPPAR-LBD) of the respective PPAR subtype. $5 \mathrm{~h}$ after transfection, medium was changed to DMEM without phenol red and 10\% FCS, supplemented with $1 \%$ SP, $1 \%$ PS and $1 \%$ L-glutamine, now additionally containing $0.1 \%$ DMSO and the respective test compound or 0.1\% DMSO alone as untreated control. Each concentration was tested in triplicate wells and each experiment was repeated independently at least three times. Following overnight incubation with the test compounds, cells were assayed for luciferase activity using Dual-Glo ${ }^{\text {TM }}$ Luciferase Assay System (Promega) according to the manufacturer's protocol. Luminescence was measured using a microplate reader (Infinite M200, Tecan Group Ltd., Crailsheim, Germany). Normalization for transfection efficacy and cell growth was done by division of the Firefly luciferase data by Renilla luciferase data resulting in relative light units. Activation factors were obtained by dividing by DMSO control. $\mathrm{EC}_{50}$ and standard deviation values were calculated by mean values of at least three determinations by SigmaPlot 2001 (Systat Software GmbH, Erkrath, Germany) using a four-parameter logistic regression. All compounds were evaluated by comparison of the achieved maximum effect to that of the reference compound (pioglitazone for PPAR $\gamma$, GW7647 for PPAR $\alpha,{ }^{25}$ and L165041 for PPAR $\delta^{26}$ each at $\left.1 \mu \mathrm{M}\right)$. Data are expressed as mean $\pm \mathrm{SE}$; $n \geq 3$.

\section{SEH activity assay}

The $\mathrm{IC}_{50}$ values of the compounds were determined by a fluorescence-based assay system of 96-well format. As substrate non-fluorescent PHOME (3-phenyl-cyano-(6-methoxy-2naphthalenyl)methyl ester-2-oxirane-acetic acid, Cayman Chemicals) was used, which can be hydrolyzed by the SEH to the fluorescent 6-methoxynaphtaldehyde. ${ }^{27}$ The formation of the product was measured $\left(\lambda_{\mathrm{em}}=330 \mathrm{~nm}, \lambda_{\mathrm{ex}}=465 \mathrm{~nm}\right)$ by a Tecan Infinite F200 Pro plate reader. Therefore, recombinant human sEH ( $2 \mu \mathrm{g}$ per well) in Bis-Tris buffer $\mathrm{pH} 7$ with 0.1 $\mathrm{mg} \mathrm{ml} \mathrm{m}^{-1}$ BSA containing a final concentration of $0.01 \%$ Triton-X 100. $100 \mu \mathrm{l}$ of protein were incubated with different concentrations of compounds (DMSO with final concentration of $1 \%$ ) for $30 \mathrm{~min}$. at room temperature. After that $10 \mu \mathrm{l}$ of substrate were added (final concentration $50 \mu \mathrm{M}$ ). The hydrolysed substrate was measured for $30 \mathrm{~min}$ (one point every minute). A blank control (no protein and no compound) as well as a positive control (no compound) was executed. All measurements were performed in triplicates. 


\section{Differentiation of murine 3T3-L1 cells}

3T3-L1 cells were subcultured in DMEM containing 10\% newborn calf serum in a humidified atmosphere at $37{ }^{\circ} \mathrm{C}, 5 \% \mathrm{CO}_{2}$. Cells were differentiated into adipocytes for 14 days according to the method of Zebisch et al. ${ }^{28}$ Briefly, cells were seeded in 6-well plates $\left(2.5 \times 10^{6}\right.$ per well). Differentiation was started at day 3 by addition of $1 \mu \mathrm{g} \mathrm{ml}^{-1}$ insulin, $0.25 \mu \mathrm{M}$ dexamethasone and $0.5 \mathrm{mM}$ isobutylmethylxanthine in DMEM supplemented with $10 \%$ fetal calf serum. At day 5 medium was replaced by medium containing only insulin for 2 more days. After this, cells were kept for lipid droplet accumulation in basal medium without additions until day 15. Rosiglitazone $(2 \mu \mathrm{M})$ and $N$-cyclohexyl- $N^{\prime}$-(iodophenyl)urea (CIU) $(10 \mu \mathrm{M})$ were used as PPAR $\gamma$ and SEH positive controls, respectively. Differentiation of 3T3-L1 cells was confirmed by Oil Red O staining. Cells were washed with PBS and subsequently fixed for 60 minutes with a formaldehyde solution (4\% in PBS). After this, cells were rinsed with $60 \%$ isopropanol and incubated with Oil Red $\mathrm{O}$ solution $(0.3 \%)$ for 120 minutes.

\section{Conclusions}

In this study diverse scaffolds of SEH inhibitors and PPAR agonists were fused in order to obtain a dual sEH/PPAR modulator. All three designed compound classes displayed potent inhibitory activity against sEH. Obviously, inhibitory activity against sEH can be easily introduced in a multi-target compound by incorporating a secondary urea or $\mathrm{N}$-benzyl benzamide fragment, as it was previously shown by Hwang et $a .^{29}$ and Meirer et al. ${ }^{30}$ However, this does not hold true for PPAR activation. Although all compounds corresponded to the general pharmacophore of PPAR agonists, ${ }^{31}$ only 8d displayed the desired PPAR modulatory activity. Even small changes in the linker region of bezafibrate displayed in $3 \mathbf{c}$ and 3d led to complete loss of PPAR agonistic properties. This observation is in line with our previous observation on dual sEH/PPAR modulators, where almost all members of a combinatorial library displayed SEH inhibitory activity, whereas only few compounds were indeed able to activate PPAR $\gamma$ and PPAR $\alpha .{ }^{14}$ Small $N$-benzyl benzamides were previously identified as a merged pharmacophores of dual sEH/ PPAR $\gamma$ modulators with oral activity, however without impact on PPAR $\alpha^{32}$ Thus, it seems to be difficult to design-in SEH inhibitory activity in a dual PPAR $\alpha / \gamma$ agonist.

The triple active agent $\mathbf{8 d}$ resulting from this study is an interesting tool for the investigation of multi-target compounds for MetS treatment. Due to the fact that it comprises SEH inhibitory and PPAR $\alpha / \gamma$ partial agonistic properties, it might simultaneously reduce dyslipidaemia, hyperglycaemia, and hypertension. With its partial agonistic profile on both PPAR subtypes, 8d should also be less prone of the typical TZD side effects including weight-gain and edema causing fluid retention. Further in vivo evaluation of $\mathbf{8 d}$ will provide valuable insights into multi-target treatment options for MetS.

\section{Acknowledgements}

This work was supported by the Else-Kröner-Fresenius Foundation graduate school Translational Research Innovation Pharma (TRIP) and Deutsche Forschungsgemeinschaft (DFG; Sachbeihilfe PR1405/1-2; SFB 1039 Teilprojekt A07). R. B. and O. D. thank the graduate school Translational Research Innovation Pharma (TRIP) for PhD scholarships.

\section{Notes and references}

1 E. S. Ford, W. H. Giles and W. H. Dietz, JAMA, 2002, 287, 356-359.

2 R. H. Eckel, S. M. Grundy and P. Z. Zimmet, Lancet, 2005, 365, 1415-1428.

3 S. M. Grundy, Nat. Rev. Drug Discovery, 2006, 5, 295-309.

4 D. V. Rollason and N. Vogt, Drugs Aging, 2012, 20, 817-832.

5 M. G. Katselou, A. N. Matralis and A. P. Kourounakis, Curr. Med. Chem., 2014, 21, 2743-2787.

6 J. D. Imig, K. A. Walsh, M. A. Hye Khan, T. Nagasawa, M. Cherian-Shaw, S. M. Shaw and B. D. Hammock, Exp. Biol. Med., 2012, 237, 1402-1412.

7 C. Duval, M. Müller and S. Kersten, Biochim. Biophys. Acta, Mol. Cell Biol. Lipids, 2007, 1771, 961-971.

8 C. Lamers, M. Schubert-Zsilavecz and D. Merk, Expert Opin. Ther. Pat., 2012, 22, 803-841.

9 I. Fleming, Pharmacol. Rev., 2014, 66, 1106-1140.

10 J. Wray and D. Bishop-Bailey, Exp. Physiol., 2008, 93, 148-154.

11 B. M. De Taeye, C. Morisseau, J. Coyle, J. W. Covington, A. Luria, J. Yang, S. B. Murphy, D. B. Friedman, B. B. Hammock and D. E. Vaughan, Obesity, 2010, 18, 489-498.

12 Y. Liu, Y. Zhang, K. Schmelzer, T.-S. Lee, X. Fang, Y. Zhu, A. A. Spector, S. Gill, C. Morisseau, B. D. Hammock and J. Y.-J. Shyy, Proc. Natl. Acad. Sci. U. S. A., 2005, 102, 16747-16752.

13 D. Xu, B. B. Davis, Z. Wang, S. Zhao, B. Wasti, Z. Liu, N. Li, C. Morisseau, N. Chiamvimonvat and B. D. Hammock, Int. J. Cardiol., 2013, 167, 1298-1304.

14 E. Buscató, R. Blöcher, C. Lamers, F.-M. Klingler, S. Hahn, D. Steinhilber, M. Schubert-Zsilavecz and E. Proschak, J. Med. Chem., 2012, 55, 10771-10775.

15 G. A. Gomez, C. Morisseau, B. D. Hammock and D. W. Christianson, Biochemistry (Moscow), 2004, 43, 4716-4723.

16 T. Asaki, T. Aoki, T. Hamamoto, Y. Sugiyama, S. Ohmachi, K. Kuwabara, K. Murakami and M. Todo, Bioorg. Med. Chem., 2008, 16, 981-994.

17 P. L. Podolin, B. J. Bolognese, J. F. Foley, E. Long, B. Peck, S. Umbrecht, X. Zhang, P. Zhu, B. Schwartz, W. Xie, C. Quinn, H. Qi, S. Sweitzer, S. Chen, M. Galop, Y. Ding, S. L. Belyanskaya, D. I. Israel, B. A. Morgan, D. J. Behm, J. P. Marino, E. Kurali, M. S. Barnette, R. J. Mayer, C. L. BoothGenthe and J. F. Callahan, Prostaglandins Other Lipid Mediators, 2013, 104-105, 25-31.

18 M. Nomura, T. Tanase, T. Ide, M. Tsunoda, M. Suzuki, H. Uchiki, K. Murakami and H. Miyachi, J. Med. Chem., 2003, 46, 3581-3599. 
19 M. Ohashi, I. Nakagome, J.-I. Kasuga, H. Nobusada, K. Matsuno, M. Makishima, S. Hirono, Y. Hashimoto and H. Miyachi, Bioorg. Med. Chem., 2012, 20, 6375-6383.

20 D. Merk, M. Zettl, D. Steinhilber, O. Werz and M. SchubertZsilavecz, Future Med. Chem., 2015, 7, 1597-1616.

21 C. Lamers, M. Dittrich, R. Steri, E. Proschak and M. Schubert-Zsilavecz, Bioorg. Med. Chem. Lett., 2014, 24, 4048-4052.

22 G. Navarrete-Vázquez, H. Torres-Gómez, S. HidalgoFigueroa, J. J. Ramírez-Espinosa, S. Estrada-Soto, J. L. Medina-Franco, I. León-Rivera, F. J. Alarcón-Aguilar and J. C. Almanza-Pérez, Bioorg. Med. Chem. Lett., 2014, 24, 4575-4579.

23 H. E. Xu, M. H. Lambert, V. G. Montana, K. D. Plunket, L. B. Moore, J. L. Collins, J. A. Oplinger, S. A. Kliewer, R. T. Gampe, D. D. McKee, J. T. Moore and T. M. Willson, Proc. Natl. Acad. Sci. U. S. A., 2001, 98, 13919-13924.

24 O. Rau, M. Wurglics, A. Paulke, J. Zitzkowski, N. Meindl, A. Bock, T. Dingermann, M. Abdel-Tawab and M. SchubertZsilavecz, Planta Med., 2006, 72, 881-887.

25 P. J. Brown, L. W. Stuart, K. P. Hurley, M. C. Lewis, D. A. Winegar, J. G. Wilson, W. O. Wilkison, O. R. Ittoop and T. M. Willson, Bioorg. Med. Chem. Lett., 2001, 11, 1225-1227.
26 J. Berger, M. D. Leibowitz, T. W. Doebber, A. Elbrecht, B. Zhang, G. Zhou, C. Biswas, C. A. Cullinan, N. S. Hayes, Y. Li, M. Tanen, J. Ventre, M. S. Wu, G. D. Berger, R. Mosley, R. Marquis, C. Santini, S. P. Sahoo, R. L. Tolman, R. G. Smith and D. E. Moller, J. Biol. Chem., 1999, 274, 6718-6725.

27 N. M. Wolf, C. Morisseau, P. D. Jones, B. Hock and B. D. Hammock, Anal. Biochem., 2006, 355, 71-80.

28 K. Zebisch, V. Voigt, M. Wabitsch and M. Brandsch, Anal. Biochem., 2012, 425, 88-90.

29 S. H. Hwang, K. M. Wagner, C. Morisseau, J.-Y. Liu, H. Dong, A. T. Wecksler and B. D. Hammock, J. Med. Chem., 2011, 54, 3037-3050.

30 K. Meirer, C. B. Rödl, J. M. Wisniewska, S. George, A.-K. Häfner, E. Buscató, F.-M. Klingler, S. Hahn, D. Berressem, S. K. Wittmann, D. Steinhilber, B. Hofmann and E. Proschak, J. Med. Chem., 2013, 56, 1777-1781.

31 H. Zettl, R. Steri, M. Lämmerhofer and M. SchubertZsilavecz, Bioorg. Med. Chem. Lett., 2009, 19, 4421-4426.

32 R. Blöcher, C. Lamers, S. K. Wittmann, D. Merk, M. Hartmann, L. Weizel, O. Diehl, A. Brüggerhoff, M. Boß, A. Kaiser, T. Schader, T. Göbel, M. Grundmann, C. Angioni, J. Heering, G. Geisslinger, M. Wurglics, E. Kostenis, B. Brüne, D. Steinhilber, M. Schubert-Zsilavecz, A. S. Kahnt and E. Proschak, J. Med. Chem., 2016, 59, 61-81. 